\title{
Performance Analysis of Blockchain based Smart Grids with Ethereum and Hyperledger Implementations
}

\author{
Hamid Malik*, Ahsan Manzoor ${ }^{\dagger}$, Mika Ylianttila $^{\ddagger}$, Madhusanka Liyanage*§ \\ ${ }^{* \ddagger \S}$ Centre for Wireless Communications, University of Oulu, Finland \\ ${ }^{\dagger}$ Rovio Entertainment Company, Espoo, Finland \\ ${ }^{\S}$ School of Computer Science, University College Dublin, Ireland \\ Email: *hamid.malik@ student.oulu.fi, †ahsan.manzoor@ rovio.com, ${ }^{\ddagger}$ [firstname.lastname]@ oulu.fi, ${ }^{\S}$ madhusanka@ucd.ie
}

\begin{abstract}
Smart grids lay the foundation for future communities. Smart homes, smart buildings, smart streets, and smart offices are built when intelligent devices piles on intelligent devices. To reach the maximum capacity, they all must be supported by an intelligent power supply. For optimal and real-time electricity consumption, monitoring and trading, blockchain posses several potential benefits in its application to electricity infrastructure. To analyze the performance of the blockchain-based smart grid, this paper presents a virtual smart grid. A smart grid equipped with smart contracts, capable of executing virtual activities is evaluated and possible strengths and weaknesses are discussed. The paper draws a performance analysis of the blockchain-based smart grid by using the Ethereum and Hyperledger Fabric-based implementations.
\end{abstract}

Index Terms-Smart Grid. Blockchain, Smart Contract, Ethereum, Hyper Ledger, Scalability, Performance

\section{INTRODUCTION}

Traditionally built smart gird infrastructure is not capable of addressing numerous challenges. As the world leaps forward towards innovation and technology, the transition from the centralized control system to a decentralized control system is of paramount importance [1].

The introduction of the Internet of Things (IoT) and smart cities with smart homes is going to revolutionize the entire digital world [2]. As we move towards the future of smart cities, smart girds play a viable role in building a digital paradise. A resilient power grid not only controls data and energy flow but also provides a backbone for a more secure, fast and scalable grid platform. Smart meters, bidirectional energy flow and digital platforms for the decentralized market mechanism are few of the many features which can potentially be handled by smart grids [3]. Smart grids equipped with these attributes will then be able to benefit prosumers and consumers with more sophisticated energy monitoring and optimized energy flow and trading.

Due to the rapid increase in renewable energy resources, there is an essential need for the evolution of the traditional energy distributed system into more resilient and smartly optimized energy grids. One of the key elements of a smart power grids is to introduce peer to peer energy ( $\mathrm{P} 2 \mathrm{P})$ trading platform [4]. For optimal and real-time electricity consumption, monitoring, and scheduling, to provide utilities to offer smart services, Smart Meter (SM) is installed. Advanced Metering Infrastructure (AMI) is one of the crucial aspects of smart grids. Smart meters are deployed to monitor and control the bidirectional energy flow and to perform secure energy transactions [5].

Ledgers have been in use for centuries and have been proven to be a very efficient way of recording and accounting transactions. With blockchain technology, the concept of the digital immutable ledger is a game-changer in the financial sector. Smart grids using blockchain matches perfectly with the attributes required for the decentralized energy market. With the help of blockchain, getting rid of the central entity and distributing power among the stakeholders, opens the possibility of a different trust model. Blockchain offers a decentralized, unaltered, shared platform. A blockchain-based smart grid, governed by self-executing codes has the potential to transform the conventional energy system structure into a more flexible and distributed one [6].

P2P energy trading over a blockchain can further lead to more benefits such as user preference energy sources, empowerment to stakeholders rather than a central authority, consensual market mechanism and increase in the overall capacity which can further counter demand response, power outages and other power grid complications.

Our contribution: To build decentralized and consensusdriven smart gird based on blockchain technology, we propose an architecture, that uses blockchain protocols as the foundation layer. This paper is providing an analysis of the performance of a virtual smart gird capable of sustaining an autonomous decentralized energy market. A smart grid using blockchain as an intermediary mostly results in a more complicated platform than conventional approaches. The creation of blocks and overall delay time adds up to unaffordable circumstances.

In this paper, we draw comparisons between two blockchain protocols, Ethereum, and Hyperledger Fabric and address the notable trade-offs to develop blockchain-based smart gird. 
The understanding of the energy market is discussed and performance evaluation of the blockchain-based smart grid is attempted to understand potential advantages and problems. We also present a smart contract-based virtual smart gird capable of performing activities like the market mechanism and payment function. Our paper presents virtual smart gird, first on public Ethereum blockchain and later on permissioned Hyperledger Fabric v1.2.

The remainder of this paper includes Section II which provides the background and explaining some of the technological concepts. The proposed architecture is explained in Section III. Implementation and comparison results are presented in Section IV. Section V discusses the limitations and analysis. Finally, Section VI presents our conclusions.

\section{BACKGROUND}

\section{A. Blockchain}

Blockchain is a special kind of data structure that performs and stores transactions in blocks. Each block is connected to a previous block with a timestamp and a hash link. Blocks provide unaltered data records and cannot be changed retrospectively. It is to be considered as a distributed kind of database where entities that do not trust each other and where there is no single point of control, can rely on each other completely decentralized [7]. Since the blocks cannot be altered without modifying the complete chain of blocks which makes the system more resilient against potential attacks [8].

During digital events among the participating entities, the blockchain acts as a public ledger of all transactions. The process of creating a new block is called mining. A consensus is held among all the participants to verify each block which ensures systems integrity and trustworthiness. Blockchain enables a distributed platform between the participating entities where the consensus is held in a democratic way [9]. Blockchain protocols can be classified into the following types.

Public blockchain: A blockchain where everyone has read access to the blockchain and may submit transactions to it such as Bitcoin and Ethereum [10].

Private blockchain: A blockchain, in which entities included in a predefined list can read the blockchain and submit transactions to it, such as Hyperledger Fabric [10].

Permissionless blockchain: A blockchain in which there are no restrictions to who is allowed to create blocks [11].

Permissioned blockchain: A blockchain in which only identities included in a predefined list are allowed to create blocks [10].

\section{B. Cryptocurrency}

Cryptocurrency is a digital asset designed for peer-to-peer digital exchange systems. A distributed aspect of blockchain which allows to generate and distribute currency units [12]. Digital cash was the first blockchain-based decentralized application, originated under the name Bitcoin which exists in the form of digital cash and also known as cryptocurrency. In 2008. the idea of blockchain along with Bitcoin was introduced by an unknown identity under the pseudonym Satoshi
Nakamoto [7]. He published the Bitcoin white paper which laid the foundation for peer to peer electronic cash systems. Since 2009 Bitcoin and other similar digital payment methods have appeared under the realm of blockchain technology.

\section{Ethereum}

Ethereum was the first blockchain-based protocol to introduce smart contracts in 2015 [12]. It provides an interactive platform by using an abstract foundation layer to deploy smart contracts written with a built-in Turing-complete programming language to develop secure, cost-efficient and smart applications with user-defined rules. Ethereum provides accounts to check current balance, to store or execute any contract within a 20-byte account address size. Ether is the digital cash and smart contracts are autonomous agents for transactions among accounts [13].

Smart contract code runs on EVM (Ethereum virtual machine) to ensure the execution environment for the virtual smart grid. EVM creates full nodes to validate new blocks. The average block generation time in Ethereum is $15 \mathrm{sec}-$ onds approximately. To prevent any cyberattack, Ethereum uses transaction fees also identified as gas consumption. The Ethereum transaction, in other words, is the amount of gas consumed multiply by gas price [13].

\section{Hyperledger Fabric}

The Hyperledger Fabric is a private and permissioned blockchain under the Linux Foundation [14]. Fabric network was first implemented in 2015 as a high confidential network on which members can track, exchange and interact with digitized assets [14]. Fabric is the first ledger to support the execution of distributed applications written in standard programming languages, known as chaincodes, which is the piece of code that is installed onto the network of Hyperledger Fabric nodes [14].

The Hyperledger Fabric architecture is comprised of the following components: Peer nodes, Endorsing Nodes, Ordering nodes, Client applications. Peers are not limited to a single role. A peer may be an endorser for certain types of transactions and just a committer for others. The work done by peer and ordering nodes is roughly the same kind of work that miners do in the other blockchain architecture [14].

\section{BlockChain BASEd SMART GRID ARChiteCtURE}

Recently, various techniques have been proposed to evaluate smart grid performance using blockchain. In Figure 1, we illustrate the general architecture of a blockchain-based smart grid.

\section{A. Distribution system operators (DSOs)}

DSOs act as mediators and are responsible for activities like market mechanisms, payment function for both consumers and local energy providers. Based on the data from corresponding blockchain accounts (consumers/prosumers), DSOs ensures private data protection and installing and maintenance of Smart Meters. DSO act as a miner to ensure consensus throughout the energy market. 


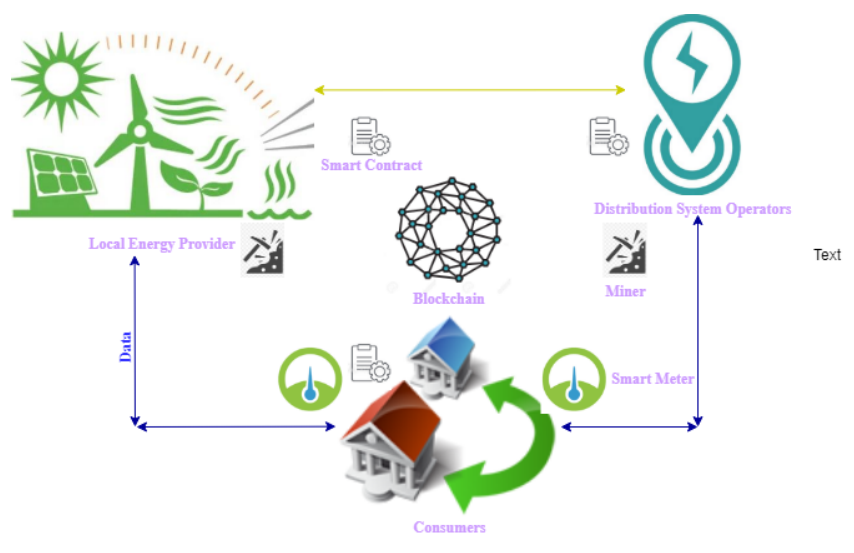

Fig. 1. Blockchain based Smart Grid Architecture

\section{B. Local energy providers}

Local energy providers produce energy using solar, wind, biogas, etc and inject energy into the smart grid. Local energy providers also act as miners in the blockchain to ensure the consensus and hence implementing the concept of the decentralized market. The mechanism is based on micropayment, the amount of energy injected is correspondingly rewarded with specified tokens using smart contracts which allows transactions over the blockchain network when certain conditions are met.

\section{Consumers}

Consumers pay the market price and act as nodes in the blockchain layer. Smart Meters are installed in every house. Smart Meter readings are recorded over the blockchain network using smart contracts. Consumers can buy electricity using specified tokens either from local energy provider or DSO.

The following steps are illustrated in Figure 1.

1) DSO deploys a blockchain-based network and installs Smart Meters with smart contracts.

2) Consumers use smart contracts to register and creates and account over the blockchain-based network. (using smart contract)

3) Smart Meter data is sent over the blockchain network and cannot be altered. (Blue lines)

4) Local energy providers along with DSOs act as miners to ensure integrity, hence achieving a decentralized market mechanism with no central authority.

5) Token-based trading system is used for buying and selling of energy. (Golden lines)

6) DSO is also responsible for delay-tolerant data like monthly bills etc.

\section{IMPLEMENTATION ON ETHEREUM}

\section{A. Architecture}

This section provides the implementation details of the Blockchain-based smart gird using Ethereum. The proposed architecture demonstrates the system design, with the smart grid implementation over a permissionless Ethereum blockchain. Figure 2 illustrates the setup of the system with a DSO, three local energy providers as three miners and three Ethereum full nodes as consumers.
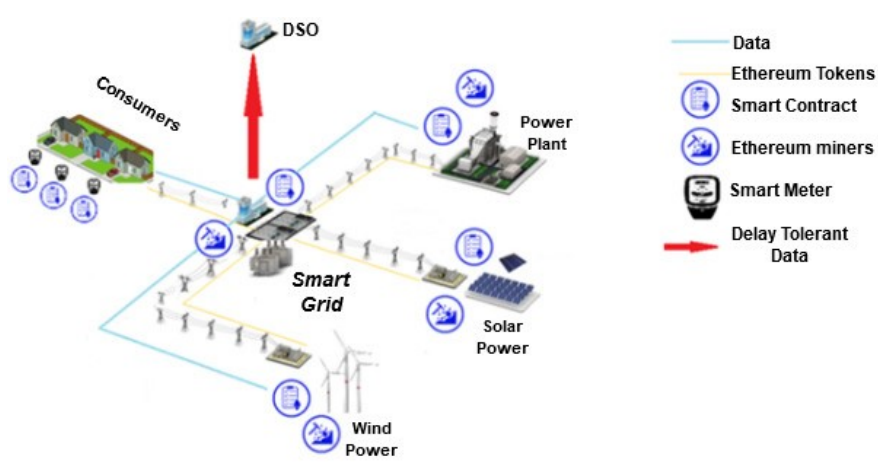

Fig. 2. Blockchain-based Smart Grid using Ethereum

\section{B. Miners and Consensus}

Figure 2 illustrates the different types of local energy providers such as solar, wind, and biogas. Local energy providers are prosumers and they have bi-directional energy flow. In the proposed system, the miners are Local energy providers and DSO, which generates a block of transaction in 15 seconds during the mining process, hence implementing a consensus mechanism by using Proof of Work. Mining rewards are transferred to Ethereum wallets, assigned to all the prosumers. Similarly, consumers also have wallets for their energy usage. Every transaction is stored in the blocks for consumers and prosumers and the market payment system is carried using Ethereum tokens.

\section{Smart Contract}

The energy market involves two smart contracts written in the Solidity programming language. Smart grid contract and Client Contract. The Smart gird contract contains a mapping that maps Smart Meter addresses to their owners under event NewCustomer. The second contract is Client contract, depending on the clients type, its contract will either be a prosumer or a consumer. Payment function is used for the electricity billing and getBalance function is used to view the current balance for the owners account.

\section{Performance analysis for Ethereum}

The smart contracts deployed on the Ethereum approach have the maximum amount of gas limit 3,000,000. As discussed above every transaction has a base. The transaction cost of the smart grid contract was 675649 gas and the execution cost was $\$ 0.1439$ as shown in Table 1.

\section{IMPLEMENTATION ON HYPERLEDGER FABRIC}

\section{A. Architecture}

In the second approach, we use Hyperledger Fabric v1.2 and measure the performance of the smart grid. Figure 3 is the proposed architecture for the blockchain-based virtual 


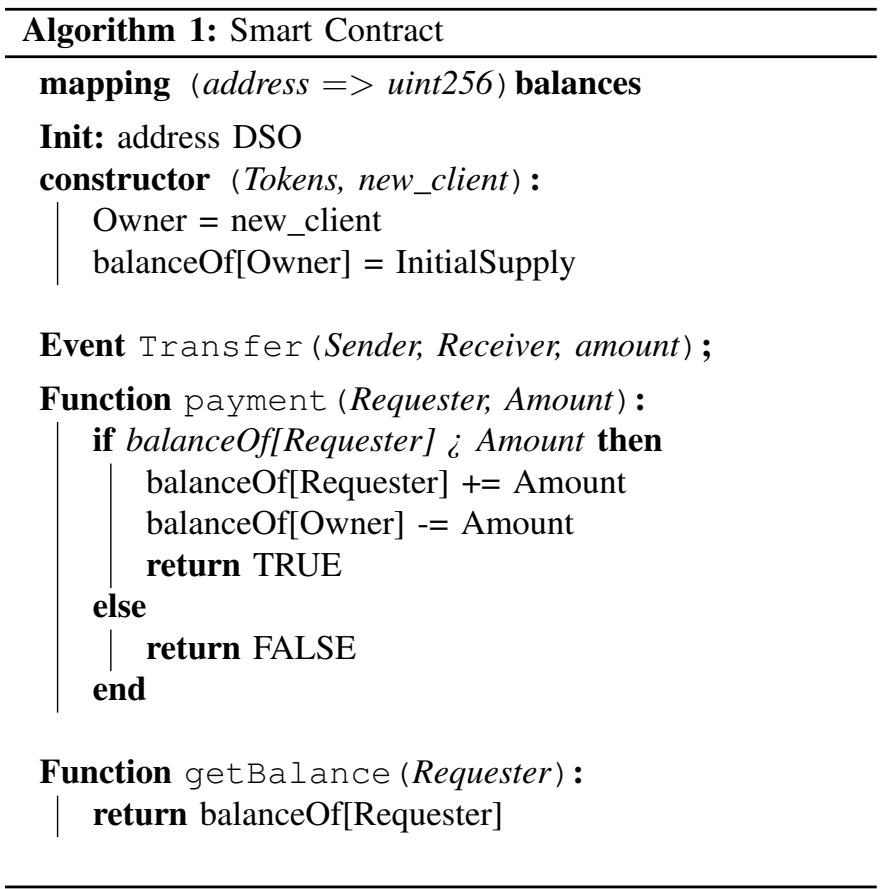

TABLE I

EXECUTION COST OF SMART CONTRACT

\begin{tabular}{ccc} 
Operation & Gas Used & Price in \$ \\
\hline $\begin{array}{c}\text { Smart grid } \\
\text { contract }\end{array}$ & 675,649 & $\$ 0.1439$ \\
\hline $\begin{array}{c}\text { Execution } \\
\text { cost Smart } \\
\text { Grid }\end{array}$ & 471,529 & $\$ 0.1004$ \\
\hline $\begin{array}{c}\text { The client } \\
\text { contract }\end{array}$ & 322,244 & $\$ 0.0686$ \\
\hline $\begin{array}{c}\text { Transaction } \\
\text { for usage of } \\
\text { electricity }\end{array}$ & 23,552 & $\$ 0.0050$ \\
\hline Get balance & 36,015 & $\$ 0.0076$ \\
\hline
\end{tabular}

smart grid using Hyperledger Fabric v1.2. The virtual grid is designed using the devices summarized in Table II.

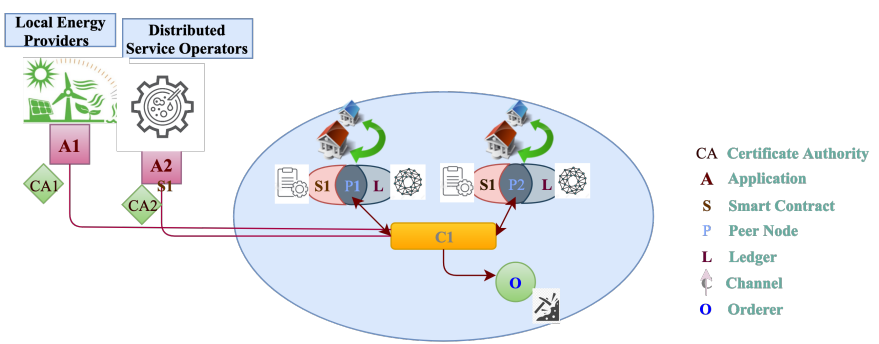

Fig. 3. Blockchain based Smart Grid using Hyperledger Fabric

\section{B. Organizations}

The proposed architecture has two organizations, DSO and Local energy providers. Each organization has its peer nodes. Organizations' role is to establish policies such as market functions and control over the grid network. Applications are associated with organizations which in our case is A1 as DSO
TABLE II

HyPERLEDGER FABRIC DEVICES

\begin{tabular}{cccc} 
& $\begin{array}{c}\text { Peers } \\
\text { Nodes }\end{array}$ & Orderer & CA \\
\hline Devices & $\begin{array}{c}\text { Virtual } \\
\text { Machine }\end{array}$ & Dell Laptop & $\begin{array}{c}\text { Virtual } \\
\text { Machine }\end{array}$ \\
\hline \# of nodes & 2 & 1 & 2 \\
\hline CPU & NA & $\begin{array}{c}2.2 \text { Intel } \\
\text { Core i5 }\end{array}$ & NA \\
\hline $\begin{array}{c}\text { \# of CPU } \\
\text { Cores }\end{array}$ & 2 & 4 & 2 \\
\hline RAM & $2 \mathrm{~GB}$ & $8 \mathrm{~GB}$ & $2 \mathrm{~GB}$ \\
\hline Hyperledger & $\mathrm{v} 1.4$ & $\mathrm{v} 1.2$ & $\mathrm{v} 1.2$ \\
\hline
\end{tabular}

and A2 as Local energy providers. The certificate authority is used for users to authenticate with respective organizations.

\section{Peers}

Peers are committers that maintain the ledger and run smart contracts to perform read/write operations. In Figure 3, the peers are the consumers and depending on client type, they are owned by either DSO or Local energy providers. The grid is extended by adding more peers.

\section{Smart Contract and Experiments}

A sample chaincode was written using Algorithm 1. It performs various transactions shown in the Calliper report in Figure 4. The code was initiated on a peer which is also connected to the channel.

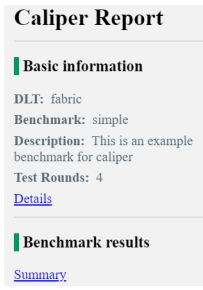

Summary

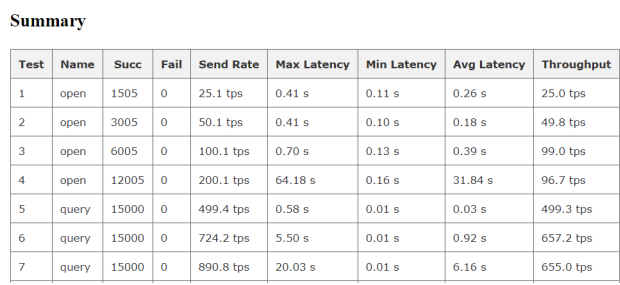

Fig. 4. Caliper Report
Unlike Ethereum, Hyperledger Fabric does not have a gas price. Hyperledger Fabric allows the application to use as much memory, storage, bandwidth and can execute as many computational steps as they want.

We perform multiple experiments to test the performance of the fabric for query transactions and invoke transactions using our sample chaincode. A machine type with 2 vCPUs and 8 GB memory, was able to execute 96.7 tps (open transactions) and 655 tps (query transactions).

\section{Discussion AND LimitaTions}

Our proposed techniques provide qualitative analysis for both implementations. Table III provides a performance analysis of both techniques based on Algorithm 1 and virtual smart gird architectures in Figure 2 and Figure 3. As we can see that Hyperledger fabric is faster and provides a flexible and configurable model. Hence the scalability issue of the Ethereum approach is tackled by proposed Hyperledger Fabric architecture. 
TABLE III

PERFormancE ANALYSIS OF VIRTUAL SMART GRID

\begin{tabular}{ccc}
\hline $\begin{array}{c}\text { Blockchain } \\
\text { type }\end{array}$ & Public & $\begin{array}{c}\text { Hyperledger } \\
\text { Fabric }\end{array}$ \\
\hline $\begin{array}{c}\text { Throughput } \\
\text { Consensus } \\
\text { mechanism }\end{array}$ & 6 & Permissioned \\
\hline $\begin{array}{c}\text { Benchmark } \\
\text { Tools }\end{array}$ & EVMremix & Calliper \\
\hline Authority & Decentralized & Centralized \\
\hline $\begin{array}{c}\text { Block } \\
\text { Generation } \\
\text { Time }\end{array}$ & 15seconds & 0.26 seconds \\
\hline Programming & Solidity & Chaincode \\
\hline Cryptocurrency & $\begin{array}{c}\text { Built-in } \\
\text { cryptocurrency }\end{array}$ & No \\
\hline
\end{tabular}

In first approach, we achieve maximum threshold of 6 transaction per second. Even if the transaction were updated every 15 minutes, only 5,000 households can be served in ideal condition which is only useful for nanogrids. To compare between two approaches, we have a significant increase from 6 tps to 96.7 tps with Hyperledger Fabric v.12 implementation. Even if the average tps is 95, more than 85,500 households can be served in 15 minutes.

The inefficiencies of the energy market and scalability were identified and fixed in this paper. There are likely more fixable inefficiencies in the virtual smart gird, that were simply not identified in this paper. Hyperledger has a significant advantage over Ethereum, regarding performance and scalability. The results have shown that Hyperledger is faster and can put up with higher throughput than Ethereum but through sacrificing decentralization.

\section{CONCLUSION}

In this paper, we have proposed blockchain-based virtual smart gird architecture which uses smart contracts for market payment and related functions. The proposed architecture was implemented by using Ethereum and Hyperledger Fabric platforms. The paper draws a performance comparison between both techniques. Ethereum is the most commonly used blockchain-based protocol but with scalability limitations. Hyperledger Fabric provides solutions to scalability but decentralization is compromised. Hyperledger Fabric is still under developing technology and potentially has the capability to solve the system limitation in smart girds.

\section{ACKNOWLEDGMENT}

This research is funded by Academy of Finland under SECUREConnect, 6Genesis Flagship (grant 318927) projects and by European Union under RE-SPONSE 5G (Grant No: 789658) project.

\section{REFERENCES}

[1] T. Hukkinen, J. Mattila, K. Smolander, T. Seppala, and T. Goodden, "Skimping on Gas-Reducing Ethereum Transaction Costs in a Blockchain Electricity Market Application," in Proceedings of the 52nd Hawaii International Conference on System Sciences, 2019.

[2] P. Porambage, J. Okwuibe, M. Liyanage, M. Ylianttila, and T. Taleb, "Survey on Multi-Access Edge Computing for Internet of Things Realization," IEEE Communications Surveys \& Tutorials, vol. 20, no. 4 pp. 2961-2991, 2018.

[3] Z. Guan, G. Si, X. Zhang, L. Wu, N. Guizani, X. Du, and Y. Ma, "Privacy-preserving and Efficient Aggregation based on Blockchain for Power Grid Communications in Smart Communities," IEEE Communications Magazine, vol. 56, no. 7, pp. 82-88, 2018.

[4] J. Abdella and K. Shuaib, "Peer to Peer Distributed Energy Trading in Smart Grids: A Survey," Energies, vol. 11, no. 6, p. 1560, 2018.

[5] Z. Zhang, Z. Qin, L. Zhu, J. Weng, and K. Ren, "Cost-friendly Differential Privacy for Smart Meters: Exploiting the Dual Roles of the Noise," IEEE Transactions on Smart Grid, vol. 8, no. 2, pp. 619-626, 2016.

[6] J.-C. Cheng, N.-Y. Lee, C. Chi, and Y.-H. Chen, "Blockchain and Smart Contract for Digital Certificate," in 2018 IEEE international conference on applied system invention (ICASI). IEEE, 2018, pp. 1046-1051.

[7] S. Nakamoto et al., "Bitcoin: A peer-to-peer Electronic Cash System," 2008.

[8] A. Panarello, N. Tapas, G. Merlino, F. Longo, and A. Puliafito, "Blockchain and IoT Integration: A Systematic Survey," Sensors, vol. 18, no. 8 , p. 2575,2018 .

[9] M. Scherer, "Performance and Scalability of Blockchain Networks and Smart Contracts," 2017.

[10] "Blockchain for Hospitality," Accessed: 11.08.2019, uRL: https://www.hospitalitynet.org/file/152008497.pdf.

[11] A. Manzoor, M. Liyanage, A. Braeke, S. S. Kanhere, and M. Ylianttila, "Blockchain based Proxy Re-Encryption Scheme for Secure IoT Data Sharing," in 2019 IEEE International Conference on Blockchain and Cryptocurrency (ICBC). IEEE, 2019, pp. 99-103.

[12] Q. K. Nguyen, "Blockchain - A Financial Technology for Future Sustainable Development," in 2016 3rd International Conference on Green Technology and Sustainable Development (GTSD). IEEE, 2016, pp. 51-54.

[13] V. Buterin et al., "A Next-generation Smart Contract and Decentralized Application Platform," white paper, vol. 3, p. 37, 2014.

[14] E. Androulaki, A. Barger, V. Bortnikov, C. Cachin, K. Christidis, A. De Caro, D. Enyeart, C. Ferris, G. Laventman, Y. Manevich et al., "Hyperledger Fabric: A Distributed Operating System for Permissioned Blockchains," in Proceedings of the Thirteenth EuroSys Conference. ACM, 2018, p. 30 